\title{
Evolución del poste muñón en Odontología
}

\section{Artículo de Revisión}

\author{
Patricia Isabel Agüero Del Carpio 1,a,b, Gerzon \\ Paredes $\mathrm{Coz}^{2, \mathrm{a}, \mathrm{b}}$, Cecilia Alayo Canales ${ }^{1, a}$ \\ ${ }^{1}$ Facultad de Odontología. Universidad Nacio- \\ nal Federico Villarreal. Lima, Perú. \\ ${ }^{2}$ Facultad de Odontología. Universidad Nacio- \\ nal Mayor de San Marcos. Lima, Perú. \\ ${ }^{a}$ Cirujano Dentista. \\ ${ }^{\mathrm{b}}$ Especialista en Rehabilitación Oral.
}

\section{Evolution of intraradicular posts in Dentistry}

\section{Resumen}

Diversos investigadores afirman que los postes brindan soporte a la restauración final en los dientes con tratamiento endodóntico, la evolución de estos ha ido desde los postes o postes de madera hasta los postes de fibra de vidrio. Esta revisión de literatura fue hecha sobre diversas bases de datos utilizando las palabras clave "técnica del poste muñón, endodoncia". Se analiza información bibliográfica relacionada a la evolución del origen de los postes con el fin de actualizar el conocimiento respecto a su uso, elección y la rehabilitación respectiva.

Palabras clave: Prótesis dental; Endodoncia; Técnica de perno muñón.

\begin{abstract}
Several researchers afirm that the posts provide support to the final restoration of teeth with endodontics, the evolution of these has gone from posts of wood to fiberglass posts. This review of the Literature was made on several databases using the keywords "post core technique, endodontics". We analyze bibliographic information related to the evolution of the origin of the posts in order to update the knowledge regarding their use, choice and the respective rehabilitation.
\end{abstract}

Keywords: Dental prosthesis; Endodontics; Post and core technique.

\section{Correspondencia:}

Patricia Isabel Agüero Del Carpio

Correo electrónico: patricia.isabel2@hotmail.com Calle Santa Bernardita 495, Pando III Etapa. Lima 01, Perú.

\section{Coautores:}

Gerzon Paredes

craneumatm2025@gmail.com

Cecilia Alayo Canales

cecilia.alayo@upch.pe

Conflicto de intereses: El autor declara no tener conflictos de interés.

Fuente de financiamiento: Ninguno.

Fecha de recepción: 01/03/17

Fecha de aceptación: 26/07/17

\section{Introducción}

Antiguamente, se pensaba que el uso de los postes servía para dar fuerza a la raíz despulpada, debido a su deshidratación y pérdida de sustancia dentinaria. Con el paso del tiempo, la tecnología, los avances científicos y los numerosos estudios de investigación, han hecho que esta afirmación cambie, debido a los persistentes fracasos en tratamientos con postes. Antes solo se podía hacer uso de los postes colados hechos de materiales que aparte de no ser estéticos, su alto módulo de elasticidad mayor a $200 \mathrm{GPa}$ contribuía a la fractura radicular del órgano dentario. La incursión de los postes prefabricados de fibras de vidrio, carbono, cuarzo y sus distintas variaciones ha mejorado sus propiedades mecánicas, físicas y estéticas de manera sustancial. Diversos repor- tes indican que las propiedades físicas y mecánicas de los postes de fibra presentan una mejor resistencia a la fractura radicular en comparación a los postes metálicos. Por este motivo es importante evaluar y considerar todos los factores necesarios para la elección de un poste adecuado dependiendo del caso. Este artículo pretende revisar la evolución del poste muñón con el fin de actualizar el conocimiento respecto a su uso.

\section{Materiales y método}

La literatura fue revisada en todos los idiomas en diversas bases de datos como Pubmed, Medline, EBSCO y SciELO publicada en los últimos 30 años, usando las palabras clave: técnica de perno muñón y endodoncia tal como aparece en los descriptores en ciencias de la salud (DeCS). Se encontraron 150 artículos, de los cuales 
se utilizaron solo 42 por tener mayor relevancia para el tema de la revisión.

\section{Características de los sustratos dentarios}

El diente está constituido por varios tejidos con diferentes índices de reflexión, refracción y absorción. La apariencia y el color de los tejidos gingivales sanos, conjuntamente con la corona, la raíz y el periodonto bańado todo a su vez por un medio líquido, se comportan como una "unidad óptica". La luz es refractada de modo casi lineal en los contornos coronarios donde hay mayor espesor de esmalte, dando así su efecto de translucidez y por el contrario, la zona central donde predomina la reflexión y la absorción, se muestra más opaca ${ }^{1}$.

Los dientes endodonciados no sólo pierden la vitalidad pulpar tras la eliminación del proceso carioso, fracturas sufridas o restauraciones anteriores, el tejido remanente queda socavado y debilitado, es por eso que se empezó hacer uso de los postes y muñones como tratamiento restaurador ${ }^{2,3}$.

\section{Evolución del perno muñón}

Las referencias más antiguas de restauraciones protésicas sobre dientes severamente destruidos, datan del periodo de Tokugawa (1603/1867) en Japón. Ellos idearon una corona con poste de madera boj, que era de color negro (estético para la época). Se utilizaba postes de madera al interior de las coronas de dientes naturales que creaba para sus pacientes sin haber hecho un tratamiento endodóntico completo, pero estos fracasaron debido a la falta de resistencia y a la absorción de humedad del medio bucal, aumentando el volumen del poste fracturando la raíz posteriormente ${ }^{2-4}$.

Pierre Fauchard ya en 1728 en su libro "Le Chirugien Dentiste ou Traité des Dens", proponía la colocación de postes estriados de oro o plata en el seno de la raíz dentaria, para retener piezas individuales o puentes fijos. Desde entonces, la profesión ha probado diferentes alternativas para la rehabilitación estética y funcional de los dientes despulpados 5 .

Claude Mounton, en 1746, diseñó una corona de oro sólidamente unida a un poste para ser insertado en el conducto radicular. Durante el siglo XIX, aparecen numerosos diseños de coronas con sistemas de anclaje radicular, pero la aportación más importante de ese siglo y en la que se basa el procedimiento actual fue la corona Richmond. Casius M. Richmond, en 1880, ideó la corona-poste constituida por tres elementos: el poste intrarradicular, el respaldo metálico y la faceta cerámica ${ }^{2}$.

Esta es una de las razones por las que el clínico que encara la restauración de un diente despulpado, habitualmente se ve enfrentado a un doble desafío: la fragilidad inherente a un diente que ha perdido su aparato nutricio e importantes estructuras arquitectónicas ${ }^{6}$ y la necesidad de reproducir las características ópticas del diente intacto, como tono, matiz, translucidez y fluorescencia ${ }^{7}$.

A partir de 1905, Taggart, gracias a la técnica de la cera perdida, logró colar metales con exactitud pudiendo así emplearlos en los postes que irían al interior de los conductos radiculares, creándose de esta manera los postes colados que daban mayor resistencia y no sufrían cambios a la humedad. Se dio un gran salto y a partir del año 1950 los postes colados se empezaron a utilizar posibilitando de esta manera colocar el poste como una restauración independiente de la corona, permitiendo así utilizar coronas cerámicas fundidas en metal en dientes remanentes con gran destrucción coronaria, además de lograr un mejor sellado de la restauración protésica ${ }^{4}$.

Al principio se utilizaban materiales nobles como la plata, pero por su elevado costo se empezaron a usar aleaciones de niquel-cromo o cromo-aluminio, estos materiales presentaban alta resistencia a la tracción, compresión y deformación, de los cuales, el último no era tan beneficioso a largo plazo debido a su alto módulo de elasticidad mayor a 200 GPa que contribuía la fractura radicular de la pieza dentaria ${ }^{2,4}$.

También fue motivo de preocupación la posibilidad de corrosión de las aleaciones metálicas nobles y no nobles empleadas para la confección de postes y/o muñones ${ }^{8}$, así como su eventual combinación con diferentes metales de incrustaciones o coronas, todo lo cual tendría el potencial de causar la fractura radicular.

Existen postes prefabricados que pueden enfatizar la retención, vía cementado, por el grabado en el metal del poste, si se usa un sistema adhesivo. De acuerdo a su superficie pueden ser activos o pasivos. Entre ellos se encuentran los postes metálicos de titanio ${ }^{4}$.

Por otro lado, el notable desarrollo logrado en la tecnología de las restauraciones libres de metal, ha llevado a la necesidad de obtener un pasaje limpio de luz que imite lo que sucede en la naturaleza ("principio de iluminación de los tejidos") ${ }^{7}$. La apariencia de la dentición natural está determinada por los efectos de la luz incidente, y el color de los dientes depende de su capacidad de modificarla ${ }^{9-11}$.

Los postes metálicos, por su opacidad, impiden el pasaje de la luz, interfieren con la natural transparencia de la encía y dan como resultado una zona oscura en el festón gingival ${ }^{12}$.

Los postes de fibra ganaron popularidad en los 90. Estos están constituidos por una matriz resinosa en la cual se encuentran inmersos varios tipos de fibras de reforzamiento. El módulo de elasticidad de los postes de fibra es similar a la dentina, poseen una menor fuerza comparada a los postes colados, son biocompatibles, se realizan en una sola sesión y muestran una mínima o nula tendencia a la fractura radicular. Además, los postes de fibra no presentan la rigidez de los postes metálicos: estas características van a hacer que este tipo de poste disipe el estrés y redistribuya las fuerzas funcionales y parafuncionales generados durante la masticación. La mayoría de postes de fibra son relativamente radiotransparentes y tienen distintos aspectos radiográficos que los postes tradicionales. Existen varias clases y marcas de postes de fibra, entre ellos están: fibra de carbono, fibra de vidrio, 
fibra de cuarzo, fibra de polietileno y fibra de cerámica con resina ${ }^{3}$.

En 1987, en Francia, apareció el primer poste de fibra de carbono, para posteriormente en 1990, ser comercializado al mercado americano. Este material innovador ofrecía un módulo de elasticidad más bajo que el de los metales o aleaciones convencionales logrando con esto un comportamiento aproximado al de la dentina lo que le daba resistencia a la raíz evitando con ello la fractura radicular. Posteriormente, diversas investigaciones que evaluaban la resistencia a la fractura demostraron que los postes de fibra de carbono eran más resistentes que los postes prefabricados metálicos y los postes colados ${ }^{13-20}$.

El color gris oscuro-negro de los postes de fibra de carbono, era una desventaja que afectaba negativamente la estética de coronas cerámicas libres de metal, según el espesor de éstas ${ }^{21-23}$. Se intentó darle solución al problema con un poste híbrido compuesto por un haz central de fibras de carbono, recubiertas con fibras de cuarzo, igualmente dispuestas en forma longitudinal que, si bien los hizo de color más favorable, no solucionó la desventaja que representa la interposición de un elemento opaco al pasaje de la luz ${ }^{24}$.

Por esta razón, la utilización de restauraciones libres de metal, inclusive hasta en espesores muy delgados y supragingivales, representa una alternativa restauradora interesante para los dientes más comprometidos del sector estético de la boca ${ }^{25}$.

Los postes de fibra de carbono se consideran estables desde el punto de vista electroquímico, es decir que, en condiciones normales, no serían pasibles de corrosión. Sin embargo, una investigación de Fovet y colaboradores probó que la misma puede producirse en determinadas circunstancias ${ }^{26}$.

Para el caso de anatomías no circulares, achatadas o con entradas de canales en forma de embudo por destrucción cariosa, se ha propuesto la realización de "postes anatómicos". Se ha denominado así a postes de fibra, generalmente translúcidos, a los que se ha modificado para adaptarlos íntimamente a la morfología del conducto, rebasándolos con resina compuesta fotopolimerizable. Luego de la polimerización se retiran, se fotopolimerizan adicionalmente y se cementan adhesivamente como si fuera un poste normal, posibilitando así espesores más adecuados del cemento ${ }^{27}$.

En 1993 fue descrito por primera vez un poste de zirconio $^{28,29}$. Se trata de un material formado por cristales tetragonales de zirconio estabilizados con óxido de itrio. $\mathrm{Su}$ principal ventaja sobre los postes estrictamente cerámicos antes mencionados, es su resistencia flexural, que es más del doble que la de aquellos y que permitiría la realización de postes de tamańos más conservadores ${ }^{30}$. Son radiopacos, fácilmente identificables en una radiografía y tienen excelentes propiedades estéticas por su capacidad de transmitir la luz de forma muy similar a las estructuras naturales ${ }^{31-33}$. De todas formas, esa ventaja se ve limitada si el propio color de la dentina radicular estuviera alterado. Además, son biocompatibles, sin actividad mutagénica ni carcinogénica ${ }^{34}$, y no presentan el problema de corrosión galvánica de los postes realizados en metales no nobles.

Pueden ser fijados con cementos clásicos y, a pesar que la recomendación ha sido utilizar preferentemente técnicas adhesivas ${ }^{32,35}$ sus valores de retención han sido significativamente menores que los de postes colados convencionales cementados con cemento de fosfato. La conclusión de los trabajos experimentales al respecto, fue que la unión entre la superficie de zirconio y la resina parece ser muy débil ${ }^{36,37}$.

Su comportamiento ha sido investigado en distintas experiencias ${ }^{38-40} \mathrm{y}$ han resultado rígidos y resistentes, pero frágiles, sin posibilidad de un comportamiento elástico mínimo y uno de los problemas que los investigadores hicieron notar, es que sus fracturas rutinariamente estaban asociadas a fracturas radiculares ${ }^{41}$.

Finalmente, Parodi afirma que la restauración de los dientes tratados endodónticamente ha representado desde siempre un desafío, tanto para el investigador como para el clínico. Algunos de los paradigmas que guiaron los procedimientos restauradores a partir de la segunda mitad del siglo pasado, han dejado paso a otras realidades, fundamentalmente gracias al impulso dado por la investigación constante que en este campo se lleva a cabo ${ }^{42}$.

\section{Conclusión}

Se debe considerar que el material del poste debe ser biocompatible y debe tener un módulo de elasticidad lo más cercano a la estructura dentaria, esto evitará las posibles fracturas de las piezas tratadas posteriormente y que necesitan ser rehabilitadas.

\section{Referencias bibliográficas}

1. Carossa S, Lombardo S, Pera P, Corsalini M, Rastello ML, Preti G. Influence of posts and cores on light transmission through different all-ceramic crowns: spectrophotometric and clinical evaluation. Int J Prosthodont 2001;14:9-14.

2. Suárez J, Ripollés M, Pradíes G. Restauración del diente endodonciado. Diagnóstico y opciones terapéuticas. Rev. Eur. Odontoestomatol. [Internet] 2006. [citado 10 Feb 2017];5:1-15. Disponible en: http://www.redoe. com/ver.php?id=42

3. Schwartz, DDS, Robbins J. Post placement and restoration of endodontically treated teeth: A literature review. J Endod. 2004;5(5):289-301.

4. Quintana M, Kobayashi A. Postes, pasado, presente y futuro. La carta odontológica. 2000;5(15):21-6.

5. Corts JP. Restauración de dientes tratados endodónticamente. En: Lanata E. y cols. Operatoria Dental. 2da ed. Buenos Aires: Alfaomega Grupo Editor; 2011.p. 283-92

6. Parodi G. Comportamiento de la dentina del diente despulpado. Factores biológicos y mecánicos. Rev Odontol Uruguaya.1995;63(1):14-20.

7. Meyenberg KH. Dental esthetics-a European perspective. J Esthet Dent. 1994;6:274-281. 
8. Aquaviva S, Shetty $S$, Coutinho I. Factors determining post selection: A literature review. J Prosthet Dent. 2003;90(6):556-562.

9. Johnston WM, Kao EC. Assessment of appearance match by visual observation and clinical colorimetry. J Dent Res. 1989;68:819-822.

10. Ten Bosch JJ, Crops JC. Tooth color and reflectance as related to light scattering and enamel hardness. J Dent Res. 1995;74:374-380.

11. Paul S. Visual and Spectrophotometric shade analysis of human teeth. J Dent Res. 2002;81;578-582.

12. Kwiatkowski SJ, Geller W. A preliminary consideration of the glass-ceramic dowel post and core. Int J Prosthodont. 1989;2:51-55.

13. Christensen J. Post concepts are changing. JADA. 2004;135:1308-1310.

14. Araujo E, Feuser L, Caldeira M; Pinos de fibra -Escolha corretamente. Arquivos em odontología. 2005;41(3):255- 262.

15. Vidyashree V. Nandini. V Venkatesh. Current concepts in the restoration of endodontically treated teeth .The Journal of Indian Prosthodontic Society. 2006;6(2):63-65

16. Quintana M, Castilla M. Restauración de piezas tratadas endodonticamente: Los postes de fibra de carbono. Rev Estomatol Herediana.1999;9(1-2):38-41.

17. Fernandes AS, Shetty S, Coutinho I. Factors determining post selection: A literature review. J Prosthet Dent 2003;90:556-562.

18. Naranjo M, Ortiz P, Osorio A, Sepúlveda J .Comportamiento de dos sistemas de postes prefabricados reconstruidos con resina sometidas a cargas cíclicas. Estudio piloto. Rev CES Odont. 2004;17(1):37-38

19. Ley A, Vera J, Dibkanan A, Henry S. Uso y abuso de los postes: Una revisión de la literatura. Rev ADM. 2002;59(4):134-136.

20. Kogan E. Postes flexibles de fibra de vidrio (técnica directa) para restauración de dientes tratados endodónticamente. Rev ADM. 2001;58(1):1-5.

21. Vichi A, Ferrari M, Davidson CI. Influence of ceramic and cement thickness on the masking of various types of opaque posts. J Prosthet Dent. 2000;83:412-417.

22. Nakamura T, Saito O, Fuyikawa J, Ishigaki S. Influence of abutment substrate and ceramic thickness on the colour of heat-pressed ceramic crowns. J Oral Rehabil. 2002;29(9):805-809.

23. Bevilaqua L, Cadenaro M, Biasotto M, Di Lenarda R, Dorigo E. Evaluation of colour differences in fiber post all-ceramic prosthetic restorations by spectrophotometry. Minerva Stomatol 2003;52:435-439.

24. Schweiger M, Frank M, Cramer von Clausbruch S, Holand W, Rheinburger V. Microestructure and properties of a pressed glass ceramic core to a zirconia post. Quintessence Dent Technol 1998;21:73-79.

25. Corts JP. Restauraciones indirectas adheridas anteriores. En: Henostroza G. Adhesión en Odontología Restaura- dora de ALODYB. 1era ed. Curitiba, Paraná, Brasil. Ed Maio. 2003. p 279 - 312.

26. Fovet Y, Pourreyron L, Gal JY. Corrosion by galvanic coupling between carbon fiber posts and different alloys. Dent Mater 2000 Sep;16(5):364-73.

27. Grandini S, Sapio S, Ferrari M. The anatomic post: an idea worth realizing. Articulo presentado en: VI Simposio Internazionale Odontoiatria Adesiva e Riconstruttiva S. Margherita Ligure 2002. Italia.

28. Lüthy H, Schärer P, Gauckler L. New materials in dentistry: zirconia posts. Abstract presentado en: IV-2 of the Monte Verità Conference on Biocompatible Materials Systems (BMS), 1993. Ascona Switzerland.

29. Sandhaus $S$, Pasche K. Tenon radiculaire en zircone pour la realisation d'inlays-cores tout ceramique. Tribune Dent 1994;2:17-24.

30. Stewardson DA. Non-metal Posts Systems. Dent Update 2001;28:326-336

31. Meyenberg KH, Lüthy H, Schärer P. Zirconia post: a new all-ceramic concept for non-vital abutment teeth. J Esthet Dent 1995;7:73.

32. Ahmad I. Yttrium-partially stabilized zirconium dioxide posts: an approach to restoring coronally compromised non-vital teeth. Int J Prosthodont 1998;18:455-465.

33. Ahmad I. Zirconium oxide post and core system for the restoration of an endodontically treated incisor. Pract Periodont Aesthet Dent 1999;11:197-204.

34. Covacci V, Bruzzese N, Maccauro G, Andreassi C, Ricci GA, Piconi $\mathrm{C}$, et al. In vitro evaluation of the mutagenic and carcinogenic power of high purity zirconia ceramic. Biomaterials. 1999;20:371-376.

35. Morgano SM, Brackett SE. Foundation restorations in fixed prosthodontics: Current knowledge and future needs. J Prosthet Dent. 1999;82:643-657.

36. Kern M, Wegner SM. Bonding to zirconia ceramic: adhesion methods and their durability. Dent Mater. 1998; 14:64-71.

37. Hedlund S, Johansson N, Sjogren G. Retention of prefabricated and individually cast root canal posts in vitro. Br Dent J. 2003;195:155-158.

38. Mannocci F, Ferrari M, Watson TF. Intermittent loading of teeth restored using quartz fiber, carbon-quartz fiber, and zirconium dioxide ceramic root canal posts. J Adhesive Dent 1999;2:153-158.

39. Akkayan B, Gülmez T. Resistance to fracture of endodontically treated teeth restored with different post systems. J Prosthet Dent. 2002;87:431-437.

40. Maccari P, Conceicao E, Nunes M. Fracture resistance of endodontically treated teeth restored with three different prefabricated esthetic posts. J Esthet Restor Dent. 2003;15:25-30.

41. Asmussen E, Peutzfeld A, Heitmann T. Stiffness, elastic limit and strength of newer types of endodontic posts. J Dent. 1999;27:275-278.

42. Parodi G,Corts J. Pernos radiculares estéticos. Evolución y aplicaciones. Actas Odontol. 2004;1(1):34-51. 